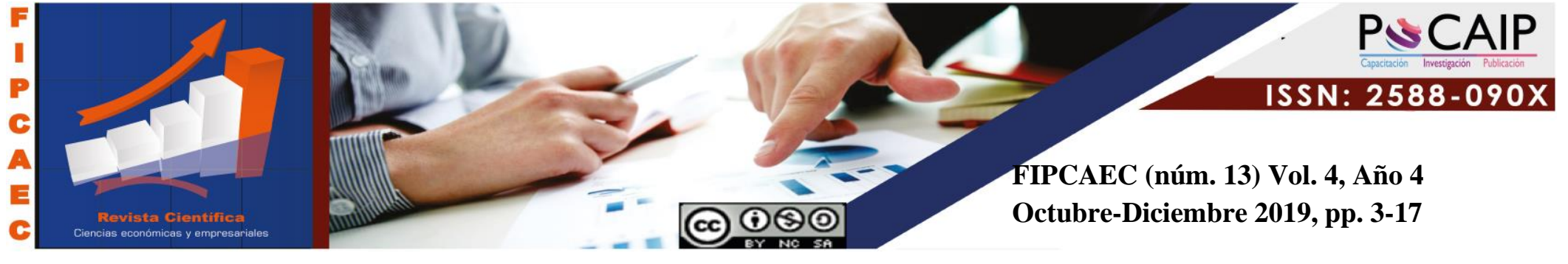

DOI: DOI 10.23857/fipcaec.v4i12.76

\title{
Cultura organizacional como alternativa a la pyme en momentos de crisis venezolana
}

\section{Organizational culture as an alternative to pyme in times of Venezuelan crisis}

\section{Cultura organizacional como alternativa às PME em tempos de crise}

\section{venezuelana}

\author{
Leslibeth Jackeline Ataco-Garcia ${ }^{1}$ \\ leslibethatacho@gmail.com \\ https://orcid.org/0000-0003-2973-6313
}

Josía Jeseff Isea-Argüelles ${ }^{2}$

josiaisea@gmail.com

https://orcid.org/0000-0001-8921-6446

Julio Juvenal Aldana-Zavala ${ }^{3}$

julioaldanazavala@gmail.com

https://orcid.org/0000-0002-7934-9103

Correspondencia: josiaisea@gmail.com

* Recepción: 12/ 07/ 2019 *Aceptación: 14/09/ 2019 *Publicación: 15 /10/ 2019

1 Doctorado en Gerencia, Magíster en Gerencia de la Calidad y Productividad, Ingeniero Químico, Red de Investigación Koinonía, Santa Ana de Coro, Venezuela.

2 Doctor en Ciencias para el Desarrollo Estratégico, Postdoctorado en Gerencia Pública y Gobierno, Post Doctorado Estado, Políticas Públicas y Paz Social, Postdoctorado en Integración y Desarrollo de América Latina, Presidente de la Fundación Koinonía, Presidente del Instituto de Estudios Avanzados Koinonía. Docente Asociado de la Universidad Nacional Experimental Francisco de Miranda, Área Ciencia de la Educación, Departamento Ciencias Pedagógicas, Santa Ana de Coro, Venezuela.

3 Doctor en Ciencias de la Educación, Postdoctoral en Estado, Políticas Públicas y Paz Social, Magíster en Orientación en Educación para Padres, Magíster Internacional en Políticas Públicas y Paz Social, Licenciado en Filosofía, Docente de la Universidad Nacional Experimental Francisco de Miranda, Área Ciencia de la Educación, Departamento Ciencias Pedagógicas, Santa Ana de Coro, Venezuela. 


\section{Resumen}

La investigación tuvo por propósito analizar la cultura organizacional como alternativa a la pyme en momentos de crisis venezolana, fundamentada desde el paradigma interpretativo, se llevó a cabo con cinco (05) integrantes que desempeñan sus funciones en diferentes niveles de la organización Caribein, S.A., los significados emergentes responden a un conjunto de elementos que consideran los valores, éticos, morales que poseen los participantes del sistema social que conforma la organización, tomando en cuenta la severa situación de crisis política, social, económica, las condiciones que afectan emocionalmente a los integrantes de la empresa, las estrategias para hacer frente a dichos inconvenientes. La cultura organizacional depende primordialmente de los valores con los que se rige la organización, siendo influenciados a través del tiempo de trabajador a trabajador, lo que define la identidad de la empresa.

Palabras clave: Empresa privada; productividad laboral; economía del trabajo; ambiente de trabajo; calidad de vida.

\section{Abstract}

The purpose of the research was to analyze the organizational culture as an alternative to SMEs in times of Venezuelan crisis, based on the interpretive paradigm, it was carried out with five (05) members who perform their functions at different levels of the organization Caribein, SA, the emerging meanings respond to a set of elements that consider the values, ethical, moral that the participants of the social system that make up the organization possess, taking into account the severe situation of political, social, economic crisis, the conditions that affect emotionally members of the company, the strategies to deal with these inconveniences. The organizational culture depends primarily on the values with which the organization is governed, being influenced through time from worker to worker, which defines the identity of the company.

Keywords: Private company; labor productivity; labor economics; work environment; quality of life. 


\section{Resumo}

O objetivo da pesquisa foi analisar a cultura organizacional como alternativa às PME em tempos de crise venezuelana, com base no paradigma interpretativo, realizado com cinco (05) membros que exercem suas funções em diferentes níveis da organização Caribein, SA, os significados emergentes respondem a um conjunto de elementos que consideram os valores éticos, morais que os participantes do sistema social que compõem a organização possuem, levando em consideração a grave situação de crise política, social e econômica, as condições que afetam emocionalmente membros da empresa, as estratégias para lidar com esses inconvenientes. A cultura organizacional depende principalmente dos valores com os quais a organização é governada, sendo influenciada ao longo do tempo de trabalhador para trabalhador, o que define a identidade da empresa.

Palavras-chave: Empresa privada; produtividade do trabalho; economia do trabalho; ambiente de trabalho; qualidade de vida.

\section{Introducción}

La cultura organizacional representa un elemento descriptivo de las organizaciones, pues de esta depende la identidad de la empresa, por cuanto proporciona los parámetros que direccionan el comportamiento de los miembros de la organización, permite el desarrollo del sentido de pertenencia en los colaboradores y favorece la integración entre los objetivos personales y los organizacionales, esto debido a que la esencia de toda organización se encuentra en su cultura, la cual es compartida por el sistema humano que la compone.

La organización es un sistema social en donde sus componentes principales son las personas, al respecto Velásquez (2013), considera que las organizaciones son unidades sociales o agrupaciones humanas constituidas para lograr metas y objetivos específicos, lo cual las hace organismos sociales, vivos y cambiantes. En el caso de las pymes, esta concepción toma especial interés, debido que al considerarse unidades productivas pequeñas, la interacción entre los integrantes se hace indispensable para el desenvolvimiento cotidiano de la empresa, siendo las 
personas las dueñas de la cultura, por cuanto en ellas reside su aceptación, promoción y desarrollo dentro del entramado social que conforma la empresa.

Teniendo en cuenta lo antes expuesto, se puede argumentar la cultura como un sistema de construcción social, según Hofstede (1991) la cultura es un constructor de sentido muy amplio, es un fenómeno colectivo compartido con otras personas, que va creciendo al compartir experiencias de vida social. La unidad social de la pyme ofrece un escenario propicio para la implantación de políticas culturales que guíen el comportamiento del talento humano hacia una organización de funcionamiento exitoso.

En este mismo sentido, Velásquez (2013) considera que a través de la cultura organizacional se fortalecen las organizaciones, por medio de las creencias básicas que construyen sus miembros como estrategia para alcanzar los objetivos planteados y enfrentar las amenazas del entorno cambiante competitivo, además agrega que la cultura organizacional "es una herramienta que permite que las personas actúen eficientemente, que tomen decisiones, que se establezcan acuerdos, modificando las estructuras tradicionales, con las que han venido operando hasta ahora". (p.95).

De la misma manera, Sánchez (2010) aporta que las organizaciones exitosas poseen culturas que atraen, retienen y recompensan al personal por desempeñar actividades y cumplir metas, con lo que considera que la efectividad de la organización se relaciona con la cultura en la motivación y maximización del valor del capital humano. En otras palabras, para garantizar el desempeño organizacional es esencial desarrollar una cultura que motive constantemente a sus integrantes.

Por lo que Sánchez (ob. cit.), opina que el conocimiento de la cultura organizacional, promueve la actuación de forma estratégica y eficiente dentro de la organización, "ya que permite que los miembros actúen en forma consistente en la organización trabajando en sentido de promover mejoras". Además agrega que la cultura organizacional es esencial para los cambios exitosos en la empresa.

Considerando el ambiente de crisis socio-económica severo que atraviesa el país, las pymes, en cuando a su capacidad de maniobra es limitada, debido a factores que escapan del control de las 
empresas, las organizaciones se encuentran en la obligación de asumir estrategias que permitan el funcionamiento a través de tiempo. Si bien, las empresas no pueden influir en el modelo económico del país, se pueden enfocar en incentivar una cultura organizacional que guíe el comportamiento de los integrantes de la pyme en pro de mantener las operaciones de la empresa, ofreciendo un producto que cumpla con las expectativas del consumidor, siendo necesario innovar tal como describe Maya (2019), por lo cual se tiene por propósito analizar la cultura organizacional como alternativa a la pyme en momentos de crisis venezolana, desde el contexto de la organización Caribein, S.A.

\section{Desarrollo}

\section{Cultura organizacional}

La cultura organizacional representa un aspecto complejo de la formación de una empresa, conlleva una gran cantidad de niveles y/o elementos que la componen, las cuales varían según la conformación organizacional atribuyendo así la identidad única de cada empresa. Ante este planteamiento es pertinente analizar los componentes de la cultura organizacional. Para López (2013) "los componentes culturales registran distintos niveles de manifestación, existiendo, por tanto, diferentes estadios de observación" (p.109), exponiendo los siguientes niveles cognitivos y de acción:

- Nivel observable, este nivel representa todos los rasgos culturales visibles de la organización, que incluye elementos como el lenguaje, los ritos, las normas, las costumbres, las conductas, las leyendas, los espacios físicos, las recompensas, las historias, los símbolos, las prácticas laborales, artilugios, entre otros.

- Nivel apreciativo y valorativo, corresponde a la parte comprehensiva de los valores, la filosofía, la ideología, las expectativas, el conocimiento, las apreciaciones, las perspectivas, las prioridades y los significados, que justifica e interpreta los elementos recogidos del nivel observable.

- Nivel fundante, este nivel engloba los elementos que integran los supuestos y creencias básicas de la organización. 
En ese mismo sentido, mencionó los niveles de manifestación cultural considerados por Schein (1988), siendo el primer nivel el de producciones, que constituye el nivel más visible de la organización, supone todo lo ofrecido por el entorno físico y social, aspectos como la capacidad tecnológica, el lenguaje, la producción artística y la conducta manifiesta, luego se tiene el segundo nivel, el cual comprende los valores por lo que el grado de visibilidad de este nivel disminuye, en este se refleja la consciencia e idea que los miembros de la organización tienen de "lo que debe ser", frente a "lo que es". El tercer nivel pertenece a las presunciones subyacentes básicas, obedece al nivel cultural más profundo, invisible e inconsciente, admite las hipótesis fundamentales del entorno, la realidad, el tiempo, el espacio, el género humano, la naturaleza de sus relaciones.

En cuanto a los elementos, Velásquez (2013) asevera que la cultura surge de la interacción de cuatro elementos básicos:

- Las creencias y valores, son aquellos principios que rigen a la organización y que considera válidos. Se refiere a las cualidades que las organizaciones desean alcanzar, que se transmiten de generación en generación, que surgen de las creencias y son la base de su implementación. La pulcritud, la disciplina, la puntualidad, el compromiso, el respeto, la responsabilidad, la honradez, entre otros.

- Los héroes; representan a los individuos que dentro de la organización encarnan y personifican las creencias y los valores, e imprimen mucho de sus características a la organización, ya que constituye una fuente de motivación e inspiración, es un símbolo, un referente, un modelo de actuación.

- Los ritos y rituales, son aquellas ceremonias simbólicas que se realizan para celebrar y reforzar valores y creencias, son ilustradas por las tradiciones y prácticas que generan emociones y que adquieren relevancia conforme transcurre el tiempo.

- Las redes culturales, este es un mecanismo oculto que forma parte de la estructura organizacional, se refiere a canales de comunicación interna e informal que se constituyen como medio básico para que los miembros de la organización interactúen y lleven a cabo sus comunicaciones. 
La cultura organizacional como las presunciones, los valores y las creencias no son observables directamente, tal como lo señala Fernández (2002), estas características deben ser inferidas mediante formas expresivas, lenguajes, ritos, mitos, normas, artefactos, entre otros, las cuales son accesibles al investigador, por lo cual se debe recurrir a las pautas de conductas y a la inquisición de los miembros de la empresa para deducir la cultura organizacional.

Como se puede observar, la cultura es un rasgo de la organización que determina el comportamiento, la estructura, así como el funcionamiento de la empresa, siendo las pymes contextos sociales más reducidos, los sentimientos, actitudes y problemas de cada individuo las afectan directamente, por cuanto las relaciones entre todos los miembros de la organización tienden a ser más estrechas y personales, con lo cual considero que los componentes culturales influyen en el ejercicio productivo, encontrándose presentes en todos los niveles de la pyme.

\section{Metodología}

La investigación se enfoca desde el paradigma interpretativo, González (2003) lo señala es el más “apropiado para estudiar los fenómenos de carácter social, al tratar de comprender la realidad circundante en su carácter específico. Mediante ella se trata de develar por qué un fenómeno ha llegado a ser así y no de otro modo" (p. 3). Cabe destacar, que este paradigma permite la comprensión de la situación del sujeto de investigación en cuanto a creencias, valores, motivaciones, entre otros, esto a través de la interpretación de la información obtenida a partir de métodos de entrevista a profundidad y observación participante.

Los procesos en la investigación cualitativa no son estandarizados, sino que la investigación evoluciona a medida que se va dando el proceso de recolección de información, es por ello que con este enfoque busco desarrollar la investigación para generar un sintagma gnoseológico gerencial sustantivo sobre la cultura organizacional, con lo que pretendo analizar, describir e interpretar las ideas, creencias, costumbres, prácticas y comportamientos que forman la cultura del sistema social de Caribein, S.A., desde la perspectiva de los informantes clave durante la interacción en el contexto de estudio. En este orden de ideas, Piñero y Rivera (2013) aseguran que la: 
....atención de los investigadores cualitativos radica en la realización de descripciones detalladas de situaciones, eventos, personas, interacciones y comportamientos observables, incorporando la voz de los participantes, sus experiencias, actitudes, creencias, pensamientos y reflexiones tal y como son expresadas por ellos mismos (p.36)

En tal sentido, se interpretó la realidad vivida desde el punto de vista del protagonista que lo experimenta, con el fin de construir a través de la reflexión una concepción del fenómeno de estudio mediante las versiones de los participantes, lo que permitió analizar la cultura organizacional ajustada a la realidad percibida durante la situación de crisis a la que está expuesta la pyme.

Se emplearon los pasos del modelo de teoría fundamentada para direccionar la obtención de la teoría sustantiva antes mencionada, al respecto Strauss y Corbin (2002) señalan que con esta metodología la teoría está fundamentada en la recolección y análisis sistemático de la información, "la teoría se desarrolla durante la investigación, y esto se lleva a cabo mediante una continua interpretación entre el análisis y la recogida de datos” (p. 273).

La investigación se llevó a cabo con cinco (05) integrantes que desempeñan sus funciones en diferentes niveles de la organización Caribein, S.A., conociéndose la opinión desde diferentes niveles de la empresa con el fin de captar la esencia cultural de la organización para la obtención de información que se ajuste a la realidad que perciben los integrantes de la empresa en momentos de crisis. 


\section{Cuadro 1}

Informantes Clave de la Investigación

\section{Código Codificación de los informantes claves}

\begin{tabular}{ll}
\hline 01GG & Gerente General \\
02EA & Encargado de Almacén \\
03PV & Promotora de Ventas \\
04ER & Encargada de Recursos Humanos \\
05OP & Encargado de Operación de Re-envasador
\end{tabular}

Nota: Los informantes clave, son sujetos pertenecientes a Caribein, S.A. una empresa del sector de las pymes ubicada en la Zona Industrial de Coro, estado Falcón, Venezuela.

\section{Resultados}

La información recabada permitió a través del análisis de contenido, aislar las categorías y subcategorías presentes en los discursos de los distintos informantes clave, en el cuadro 1, se presentan la cromatización utilizada para el procesamiento de información, dichas categorías ofrecen la perspectiva cultural de la empresa durante los tiempos críticos. Cabe destacar que para selección de las categorías, siguiendo la metodología de Strauss y Corbin (2002) de teoría fundamentada, se realizó la codificación abierta, luego la codificación axial y finalmente el proceso de codificación selectiva dando origen a la determinación o surgimiento de las categorías de estudio presentes en el discurso de los integrantes de la organización.

\section{Cuadro 2}

Cromatización de las categorías

\begin{tabular}{lll}
\hline Categoría & Sub-categoría & Color \\
\hline Cultura Organizacional & Cultura en la organización & Morado
\end{tabular}

Nota: El cuadro representa la cromatización (color) asignado a cada sub categoría con la finalidad de facilitar la estructuración de la información emergente. 
El estudio se enfocó a la determinación de un sintagma gnoseológico gerencial sobre cultura organizacional dentro del contexto actual de crisis, por lo cual se aboca al desarrollo de dicha concepción a partir de la perspectiva de los participantes en el estudio, a fin de obtener una teoría sustantiva que ofrezca una herramienta a la gerencia para enfrentar la crisis socioeconómica actual.

\section{Hallazgos en la categoría medular 1: Cultura organizacional}

La categoría medular 1 correspondiente a la cultura organizacional, se subdivide en tres subcategorías presentes en el discurso de los informantes clave, siendo estas; cultura en la organización y participación del personal, la información obtenida en la codificación abierta de los informantes clave 01GG, 02EA, 03PV, 04ER, 05OP, permitió adquirir información sobre la perspectiva de los integrantes de la empresa, en la figura 1 se muestran los resultados de la codificación para esta categoría.

Figura 1. Categoría medular 1: Cultura organizacional

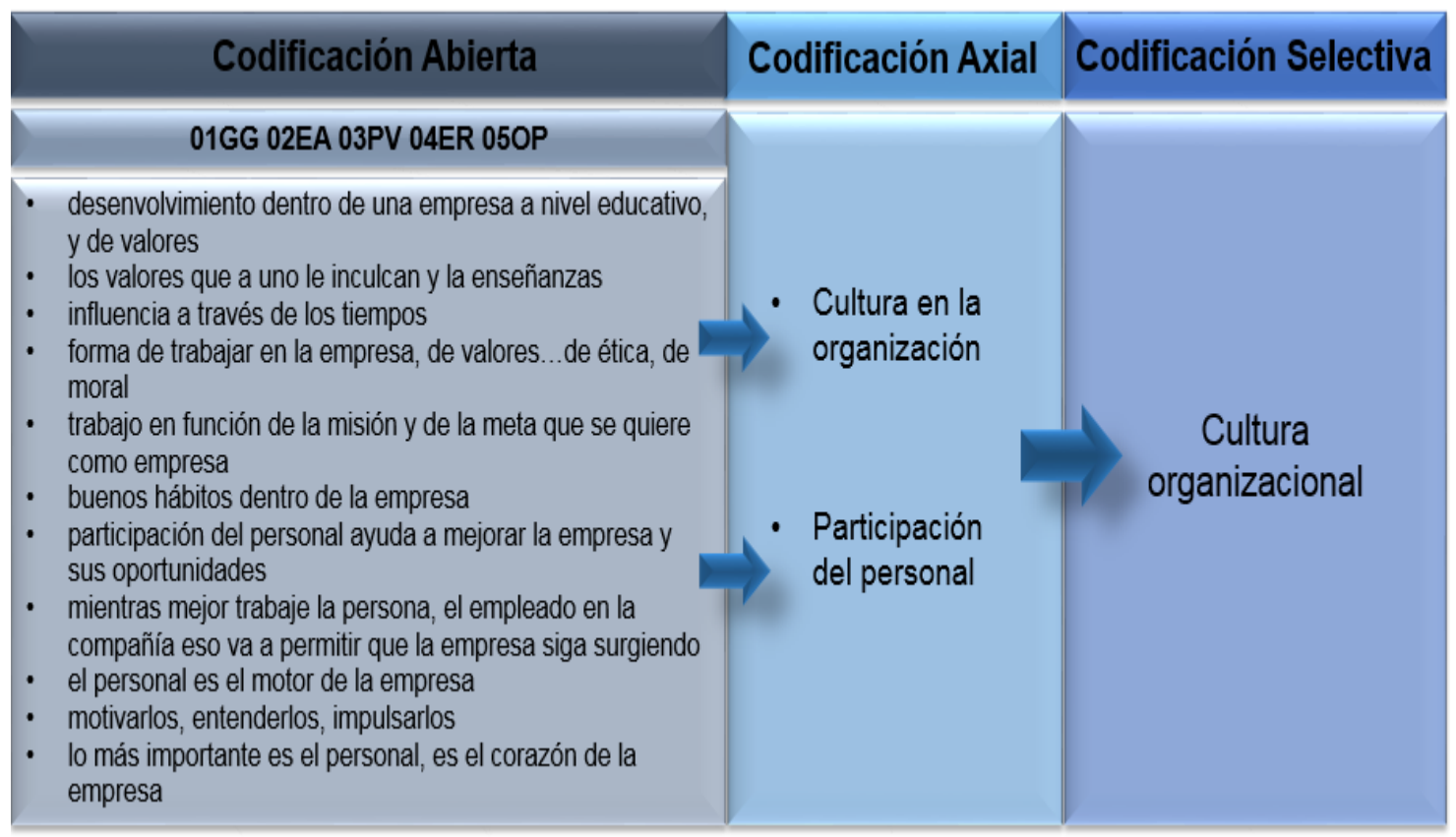




\section{Sub-Categoría: Cultura en la Organización}

En el figura 1 se puede observar que la categoría medular cultura organizacional posee dos subcategoría derivadas en la codificación axial, la primera de ellas, que engloba la percepción de los informantes clave con respecto a la cultura en la empresa donde desarrollan sus actividades, señala la influencia que los integrantes de una organización tienen, los valores éticos y morales, de ahí que para ellos la cultura se expresa principalmente a través de los valores personales. Entre los hallazgos de esta sub-categoría cultura en la organización, se encuentran:

- Desenvolvimiento dentro de una empresa u organización a nivel ecológico, educativo y de valores. 01GG-p1

- Los valores que a uno le inculcan y las enseñanzas. 02EA-p1.1

- Influencia a través de los tiempos. 02EA-p1.2

- Forma de trabajar en la empresa, de valores, de ética, de moral. 03PV-p1

- Trabajar en función de la misión...dentro de los rangos esperados o dentro de la meta que nosotros queremos como empresa. 04ER-p1

- Buenos hábitos dentro de la empresa. 05OP-p1

Al respecto se infiere que los informantes clave participantes en la organización, asumen la cultura organizacional como parte esencial en el comportamiento y clima organizacional, por cuanto según su percepción, le atribuyen a la cultura organizacional la reacción, desenvolvimiento de los integrantes en la empresa, las funciones, respuestas y desempeño en sus labores.

Todo esto evidencia la claridad en cuanto a la cultura organizacional como elemento dentro de la organización, de ahí que para Cantú (2006), la cultura en la organización responde a "sentimientos que se vive en una organización, debido al medio físico y a la forma en que interactúan sus miembros entre sí y con externos” (p. 434). De acuerdo a los postulados antes expuestos, la percepción que los integrantes de la pyme poseen con respecto a esta sub-categoría se encuentra acorde a las teorías existentes, considerando la cultura en la organización desde un punto de vista ideal, lo cual indica el nivel de conocimiento y claridad que sobre cultura se maneja entre los trabajadores de esta empresa, sin embargo, la investigación pretende dar 
respuestas a vacíos teóricos sobre la cultura organizacional en los tiempos de crisis que atraviesas las organizaciones en el país actualmente.

\section{Reflexiones de cierre}

Durante los hallazgos se buscó identificar los vacíos teóricos, al respecto se puedo determinar que las concepciones sobre cultura organizacional son realizadas a partir de un escenario ideal, mientras que el presente estudio se enfocó en considerar el escenario de crisis actual que atraviesa Venezuela desde hace unos años que es experimentado por las pymes regularmente, al cual deben hacer frente para mantener sus operaciones. Sin embargo, es necesario resaltar que para los informantes clave, la cultura organizacional depende primordialmente de los valores con los que se rige la organización, que son influenciados a través del tiempo de trabajador a trabajador, lo que define la identidad de la empresa.

No obstante, se debe mencionar que Cantú (2006) realizó un intento por definir desde una visión menos ideal la cultura organizacional, al asegurar que representa una serie de supuestos compartidos en la organización aprendidos durante la resolución de problemas y adaptación externa e integración interna, y como se puede evidenciar, es un tímido intento de conferirle a la cultura organizacional el papel regulador y direccionador que puede poseer a la hora de una crisis económica sociopolítica.

En cuanto a los significados emergentes que le atribuyen los informantes clave a la cultura organizacional en la pequeña y mediana empresa en tiempos de crisis, responde a un conjunto de elementos que consideran los valores, éticos, morales que poseen los participantes del sistema social que conforma la organización, tomando en cuenta la severa situación de crisis política, social, económica, las condiciones que afectan emocionalmente a los integrantes de la empresa, y las estrategias para hacer frente a dichos inconvenientes.

se determinó que la cultura organizacional en tiempos de crisis es una herramienta para hacer frente a las amenazas del entorno generadas por la severa crisis económica, sociopolítica que atraviesa el país, que está compuesta por el conjunto de valores, éticos, morales que guían el comportamiento y que es promovida, impulsada, aplicada por los integrantes de la empresa, 
manteniendo el optimismo y la buena disposición ante la crisis, en otras palabras, de la actuación del sistema humano de la organización depende el buen funcionamiento

\section{Referencias}

1. Fernández, J. (2002). Cultura de la organización y centro educativo. Investigación Doctoral. Universidad Complutense de Madrid. ISBN: 84-669-2288-1. Madrid España.

2. González, A. (2003). Los Paradigmas de Investigación en las Ciencias Sociales. Recuperado de https://es.scribd. com/doc/29823675/Los-paradigmas-de-investigacion-enciencias-socieles

3. Hofstede, G. (1991). Cultures and Organizations. USA: McGraw-Hill.

4. López, M. (2013). La cultura organizativa como herramienta de gestión interna y de adaptación al entorno. Un estudio de casos múltiple en empresas murcianas. Tesis Doctoral. Universidad de Murcia España.

5. Maya, K. (2019). Innovación para el desarrollo económico del Gremio Artesanal. Revista $\begin{array}{llll}\text { Arbitrada Interdisciplinaria } & \text { Koinonía, } & 4(7), & \text { 88-102. }\end{array}$ doi:http://dx.doi.org/10.35381/r.k.v4i7.195

6. Piñero, M. y Rivera, M. (2013). Investigación cualitativa: orientaciones procedimentales. UPEL-IPB, Barquisimeto Venezuela.

7. Sánchez, M. (2010). Caracterización de la cultura organizacional de las instituciones de educación superior del estado de Guanajuato. Tesis Doctoral. Universidad Autónoma de Querétaro. México.

8. Schein, E. (1988). La cultura empresarial y el liderazgo. Barcelona, España: Plaz \& Janés.

9. Strauss, A. y Corbin, J. (2002). Bases de la Investigación Cualitativa. Técnicas y Procedimientos para Desarrollar la Teoría Fundamentada. Editorial Universidad de Antioquia. Colombia 
10. Velásquez, Y. (2013). Cultura organizacional en la gestión de los institutos universitarios de tecnología y las políticas públicas que impulsa el estado venezolano. Tesis Doctoral. Universidad de Carabobo Venezuela.

\section{References}

1. Fernández, J. (2002). Culture of the organization and educational center. Doctoral Research Complutense University of Madrid. ISBN: 84-669-2288-1. Madrid Spain.

2. González, A. (2003). The Paradigms of Research in the Social Sciences. Recovered from https: //en.scribd. com / doc / 29823675 / The-paradigms-of-research-in-social-sciences

3. Hofstede, G. (1991). Cultures and Organizations. USA: McGraw-Hill.

4. López, M. (2013). The organizational culture as a tool for internal management and adaptation to the environment. A multiple case study in Murcia companies. Doctoral thesis. University of Murcia Spain.

5. Maya, K. (2019). Innovation for the economic development of the Craft Guild. Interdisciplinary Arbitrated Review Koinonía, 4 (7), 88-102. doi: http: //dx.doi.org/10.35381/r.k.v4i7.195

6. Piñero, M. and Rivera, M. (2013). Qualitative research: procedural guidelines. UPELIPB, Barquisimeto Venezuela.

7. Sánchez, M. (2010). Characterization of the organizational culture of higher education institutions in the state of Guanajuato. Doctoral thesis. Autonomous University of Queretaro. Mexico.

8. Schein, E. (1988). Corporate culture and leadership. Barcelona, Spain: Plaz \& Janés.

9. Strauss, A. and Corbin, J. (2002). Basis of Qualitative Research. Techniques and Procedures to Develop the Grounded Theory. Editorial University of Antioquia. Colombia 
